\title{
Adaptive Immune Responses and HER2/neu-Positive Breast Cancer
}

\author{
Eric D. Mortenson · Yang-Xin Fu
}

Published online: 11 December 2012

(c) Springer Science+Business Media New York 2012

\begin{abstract}
Oncogenic signaling, such as HER2/neu signaling, has been shown to play a major role in tumorigenesis in a subset of breast cancer patients. The use of anti-HER 2/neu antibody has not only revealed the mechanisms for HER2/neu signaling, but has also shown a therapeutic advantage of its blockade. The use of trastuzumab has greatly improved the treatment of HER2positive breast cancer. Although this therapy has been used in the clinic for over 20 years, recent data are still uncovering new mechanisms by which this antibody exerts its antitumor activity. In addition to improved understanding of the molecular mechanisms by which this therapy inhibits growth of tumor cells, it has been discovered that anti-HE2/neu therapy initiates and requires the adaptive immune system. The presence of anti-HER2/neuinitiated adaptive immunity gives credence to efforts targeted at stimulating the immune system in treating HER2-positive breast cancer. This review focuses on the role of the inflammatory response in HER2-positive breast cancer with particular emphasis on trastuzumab therapy.
\end{abstract}

Keywords Oncogenic signaling · HER2/neu - Adaptive immunity $\cdot$ Breast cancer $\cdot$ Antibody therapies

E. D. Mortenson · Y.-X. Fu ( $\square)$

Department of Pathology and Committee on Immunology, University of Chicago, 924 E 57th St., BSLC R102, Chicago, IL 60637, USA

e-mail: yxfu@bsd.uchicago.edu

\section{Introduction}

The use of antibody therapies has changed the landscape for treating multiple cancer types. Over the past 12 years, the FDA has approved multiple antibodies for use against human malignancies, establishing this therapy to be effective in treating cancer. In fact, antibody-based therapeutics are now essential components of many cancer treatment regimens [1]. Moreover, the success these therapies are having in the clinic is driving further development of novel immunotherapies [2]. Among the oncoproteins against which antibodies have been generated, human epidermal growth receptor factor 2 (HER2/neu/ErbB2) is among the most targeted, with two molecular targeting agents approved by the FDA [3].

The HER2 oncogene is the human homologue of the rat neu oncogene. First identified in DNA from ethylnitrosourea-induced neuroglioblastomas in rats, HER2/neu is a $185-\mathrm{kDa}$ protein with homology to epidermal growth factor receptor [4-6]. Along with HER3 (ErbB3) and HER4 (ErbB4), these proteins constitute the type 1 growth factor receptor family [7]. These transmembrane proteins are receptor tyrosine kinases that have intrinsic kinase activity and are activated when ligands bind and promote homodimerization or heterodimerization [7, 8]. However, no specific ligand for HER2/neu (ErbB2) has been identified $[8,9]$. When normally expressed, heterodimerization of HER2/neu induces downstream signals through the Akt and mitogen-activated protein kinase pathways. Signaling through these receptors supports many physiological processes, including embryogenesis, cell proliferation, differentiation, adhesion, motility, and apoptosis [10, 11].

Studies using monoclonal antibodies specific for the HER2/neu protein revealed that targeting this surface protein could block oncogenic signaling and inhibit tumor 
growth. In vitro treatment of neu-transformed fibroblasts or a human mammary gland adenocarcinoma cell line with an anti-neu antibody (clones 7.16.4 and 4D5, respectively) resulted in downregulation of surface neu protein, and growth inhibition $[12,13]$. When used to target xenografts of human tumors in nude mice, anti-neu antibody therapy inhibited tumor growth in vivo [14]. Early studies also demonstrated overexpression of HER2/neu provided transformed cells with resistance to TNF $\alpha$-mediated tumor inhibition in vitro [15]. However, treatment of these cells with anti-HER2/neu antibody countered the resistance to TNF $\alpha$ and induced tumor cell death [13].

Owing to the accessibility of HER2/neu at the cell membrane, and its low expression in normal tissues and overexpression in a high percentage of tumors, this protein was an ideal candidate for immunotherapeutic intervention [16]. Trastuzumab is a humanized monoclonal antibody that was engineered by inserting the complementary regions of the murine antibody (clone 4D5) into a human IgG1 framework [17, 18]. Since its development, trastuzumab has been tested in several clinical trials and has proved to be an effective adjuvant therapy for HER2/neupositive metastatic breast cancers [19]. In 1998, the FDA approved trastuzumab (Herceptin) for use in treating human breast cancer patients. It was the first monoclonal antibody to be approved for treating solid tumors. The therapeutic activity of trastuzumab has been evaluated in women with metastatic breast cancer as a single agent given before or after traditional chemotherapy, and in combination with a variety of chemotherapy agents [11, 20-22]. The first phase II trials demonstrated objective response rates from 12 to $15 \%$ [22, 23]. These studies established that trastuzumab therapy can be effective in patients [24]. On the basis of the convincing preclinical studies, clinical trials were conducted and demonstrated the benefits of combining chemotherapy with trastuzumab therapy [24-26]; one such study enrolled women who had not received previous adjuvant therapy to examine the combination of trastuzumab therapy and chemotherapy [20, 24]. The addition of trastuzumab therapy to chemotherapy was associated with a longer time to disease progression, a higher objective response rate, and longer survival [20]. This study was instrumental, and on the basis of these results, the FDA approved trastuzumab, given either alone or in combination with chemotherapy, for treating patients with metastatic breast cancer overexpressing HER2/neu [1,9].

\section{Immune Responses to Breast Cancer}

Multiple studies have been published regarding the mechanisms by which anti-HER2/neu therapy inhibits tumor growth. Anti-HER2/neu therapy has both direct and indirect effects that ultimately lead to tumor cell death. The direct effects include diminishing cell signaling, induction of cell cycle arrest, inhibition of receptor shedding, and when combined with chemotherapy, inhibition of DNA repair. The indirect effects involve inhibition of angiogenesis and inflammatory cell engagement. These multiple effects are due to the ability of anti-HER2/neu to prevent HER2/neu dimerization while the effector Fc arm of the antibody engages $\mathrm{Fc}$ receptor (FcR)-positive inflammatory cells, such as natural killer (NK) cells and macrophages. In fact, FcR-positive cells were shown to be essential for mediating the therapeutic effects of the anti-HER2/neu antibody [27]. A study by Clynes et al. [27] demonstrated that the therapeutic effect of the anti-HER2/neu antibody was significantly reduced in $F c \gamma R$-knockout mice. In addition, when a mutation was made in the $\mathrm{CH} 2$ domain of the mouse IgG2 heavy chain to inhibit FcR binding, antiHER2/neu maintained an ability to inhibit tumor growth in vitro, but did not induce antibody-dependent cellular cytotoxicity (ADCC) by NK effector cells. Although Fc-mediated mechanisms do not exclude other mechanisms of action by anti-HER2/neu, these data promoted ADCC as the major mechanism for the in vivo effects of anti-HER2/ neu therapy. Over the past few years, the direct effect antiHer2/neu therapy has on HER2-positive tumors has been reviewed extensively $[18,28]$. Therefore, this review will focus on new evidence for how anti-HER2/neu therapy induces an adaptive immune response.

Understanding the role of adaptive immunity in HER2positive breast cancer has been an important area of study for many years. For example, in the early 1990s, many studies demonstrated that both humoral and adaptive immune responses against HER2/neu are present in HER2/neupositive beast cancer patients [29]. However, how antiHER2/neu therapy induces this response and if adaptive immunity was even necessary was largely underappreciated. This was due in part to the overwhelming evidence that antiHER2/neu therapy exerts multiple direct effects on the tumor, and these mechanisms were observed and supported by in vivo evidence [24]. One caveat to many of these studies, however, was the use of xenografting of human tumors into nude mice. As a result, the role of adaptive immunity in anti-HER2/neu therapy was underexplored.

Using an immunocompetent mouse model, we recently demonstrated that adaptive immunity and $\mathrm{T}$ cells are necessary for tumor reduction by anti-HER2/neu therapy alone [30••]. Wild-type BALB/c mice were inoculated with a syngeneic HER2/neu-positive breast cancer cell line and treated with a mouse anti-HER2/neu antibody. The critical role of adaptive immunity was supported by the observation that the efficacy of anti-HER2/neu therapy was greatly reduced in Ragl-knockout mice that lack adaptive 
immunity. Anti-HER2/neu therapy had some effect in Rag 1knockout mice, suggesting that the direct effects of antiHER2/neu therapy were intact, but the overall antitumor effect was significantly reduced in these mice. To explore the cell types involved, wild-type BALB/c mice depleted of $\mathrm{CD} 8^{+} \mathrm{T}$ cells were unable to completely respond to anti-neu therapy, and this CD8 dependency was recapitulated in tolerized neu transgenic mice. Moreover, wild-type BALB/c mice with undetectable tumors for greater than 30 days after anti-HER2/neu therapy were subsequently resistant to a high-dose tumor rechallenge, suggesting the presence of immune memory. Together these data suggest that, in addition to the direct effects anti-HER2/neu therapy has on inhibiting tumor growth, this treatment also induces and requires an adaptive immune response.

We also investigated the inflammatory events leading to the induction of adaptive immunity after anti-HER2/neu therapy. Stimulation of Toll-like receptors (TLRs) via the MyD88 pathway is an important means by which antigenpresenting cells are activated and respond to foreign pathogens [31]. Accordingly, the antitumor effect of anti-HER2/ neu therapy was abolished in MyD88-deficient mice. Typically, TLRs are activated via pathogen-associated molecular patterns from bacteria and viruses. However, some selfligands associated with tissue damage, such as high mobility group box 1 (HMBG1), are also capable of activating TLRs to increase cross-priming and activation of dendritic cells in both mice and humans $[32,33]$. Neutralization of HMBG1 alone greatly reduced the efficacy of anti-HER $2 /$ neu therapy. These data indicate that HMGB1, an endogenous danger signal, is essential for antibody-mediated tumor regression, and it is conceivable that anti-HER2/neu antibody induces HMGB1 release in the tumor microenvironment, enhancing innate responses via the MyD88 pathway.

These observations were extended and confirmed by work from Smyth's group [34••], who-through the use of another HER2/neu-dependent tumor model and adoptive cell transfer experiments with CD8 and interferon- $\gamma$ knockout micedemonstrated that not only were $\mathrm{CD} 8^{+}$cells essential, but interferon- $\gamma$ from $\mathrm{CD}^{+}$cells was required for the antitumor effect of anti-neu therapy. Although this study also demonstrated that anti-HER2/neu therapy was dependent on TLR signaling through MyD88, it established the necessity of NK cells and the additional role of type I interferons. Taken together, these two studies suggest that NK cells induce ADCC after engaging the anti-HER2/neu antibody to promote the release of HMGB-1, stimulate MyD88-dependent TLRs, and thereby cause the release of type 1 interferons to prime the adaptive immune system (Fig. 1).

Pertuzumab is a humanized monoclonal antibody that binds extracellular domain II of the HER2 receptor, and has been effective in treating trastuzumab-resistant tumors when given in combination with trastuzumab [35]. The binding site of pertuzumab differs from that of trastuzumab, which binds extracellular domain IV, but it nonetheless shares many of the same mechanisms of action [35]. Because pertuzumab can induce ADCC, it follows that induction of an adaptive immune response would be possible. It remains to be determined, however, if adaptive immunity is necessary for pertuzumab therapy.

Although there is still much to learn about how antiHER2/neu therapy inhibits tumorigenesis and how to combine it with standard care-be it radiation, chemotherapy, or surgery-these studies identified a new mechanism of action for anti-HER2/neu therapy and support continued efforts aimed at harnessing this response for enhanced tumor reduction.

\section{Immunotherapy for Breast Cancer}

Prior to our study, other researchers had been attempting vaccination, regulatory $\mathrm{T}$ cell depletion, and adoptive cell transfer; but these immunotherapies have had limited and various effects. Despite a measurable immune response, increased numbers of tumor-specific $\mathrm{CD}^{+} \mathrm{T}$ cells do not always lead to tumor reduction. Furthermore, recognition of the tumor antigen alone is not sufficient for the host to eradicate established solid tumors [36-38]. Thus, natural immune responses fail to clear tumor cells and immune tolerance may occur throughout tumor progression. Several factors contribute to this dampened immune response. First, poor direct or indirect antigen presentation in lymphoid tissues reduces early $\mathrm{T}$ cell priming. This may be due to inadequate numbers of tumor cells migrating to the lymph node, low expression of MHC class I on tumor cells, or low expression of costimulatory molecules on antigenpresenting cells. Second, physical (e.g., hydrostatic pressure) and biological (poor adhesion molecule activation) barriers around tumor tissues can cause an inadequate number of immune cells to migrate into and though the tumor [39, 40]. Third, suppressive cell types residing within the tumor microenvironment can induce anergy or deletion of infiltrating $\mathrm{T}$ cells to promote tumor growth [41]. Fourth, release of soluble factors such as IL-10, transforming growth factor $\beta$, IL-17, and vascular endothelial growth factor by the tumor or the surrounding stroma can inhibit $\mathrm{T}$ cell function or promote angiogenesis $[38,42]$. Finally, immune tolerance and/or suppression can be induced by the tumor itself though the expression of the inhibitory molecule PD-L1.

\section{Combination Therapies}

Owing to the multitude of factors limiting the immune response, the need for therapies that reactivate the immune 


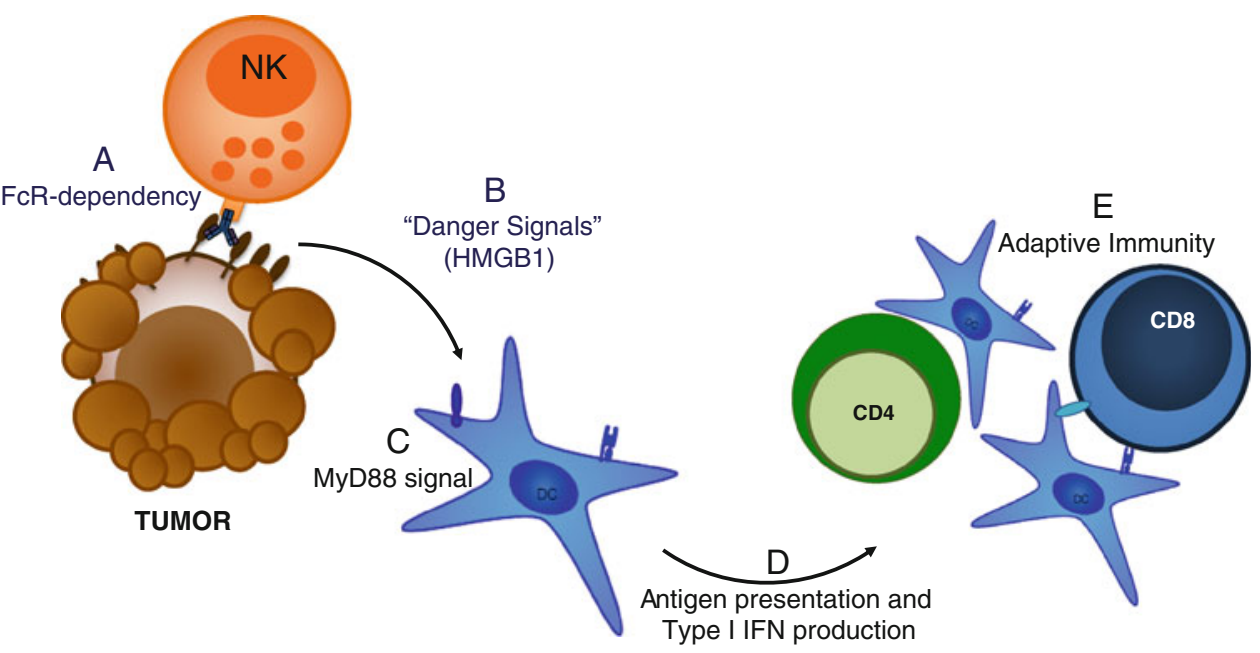

Fig. 1 Anti-HER2/neu therapy induces an adaptive immune response. Tumor cell death induced by oncogenic signal blockade and antibody-dependent cellular cytotoxicity upon binding of antiHer2/neu to the Her 2 receptor induces release of danger signals, such as high mobility group box 1 ( $H M G B 1)$, from apoptotic or stressed tumor cells $(A)$. HMGB1 is recognized alone or in complex with other molecules, such as single-stranded DNA, to activate dendritic cells and promote cytokine production via activation of the MyD88 pathway (B). MyD88 signaling enhances cross-presentation and activates dendritic cells and also induces the release of type 1 interferons $(I F N)(C)$. These dendritic cells can either remain in the tumor or travel to the lymph node and prime $\mathrm{CD}^{+}$and $\mathrm{CD}^{+}{ }^{+} \mathrm{T}$ cells of the adaptive immune system $(D)$. Primed T cells leave the lymph node and travel back to the tumor. $F c R$ Fc receptor. $N K$ natural killer cell

neu therapy when administered to both tumor-bearing wildtype mice and neu transgenic mice. In a similar fashion, Kohrt et al. [46] recently reported that CD137 expression is increased on human NK cells after anti-HER2/neu therapy, and selectively activating this protein with an agonistic antiCD137 antibody enhanced the efficacy of anti-HER2/neu therapy in a xenograft model. Transplanting human tumors into aythmic mice allowed these researchers to identify how additional anti-CD137 treatment enhances ADCC; however, how activating signals through anti-CD137 therapy enhances adaptive immune responses still requires further elucidation.

In our laboratory, we have focused on the immunostimulatory properties of LIGHT. Expression of LIGHT, a TNF family member, within the tumor microenvironment can attract various immune cells, including substantial numbers of $\mathrm{FcR}^{+}$cells, dendritic cells, and $\mathrm{T}$ cells [47]. In the context of HER2/neu-positive breast cancer, targeting tumors with an adenovirus expressing LIGHT enhanced the efficacy of anti-HER2/neu therapy [30••]. Moreover, tumor-free mice after combination therapy were resistant to a lethal rechallenge of HER2-positive tumor cells but not HER2-negative cells. These data suggest that expression of LIGHT works by enhancing the adaptive immune response and promoting antigen-specific memory. Together, these preclinical studies support further effort aimed at enhancing the adaptive immune response initiated by anti-HER $2 /$ neu therapy. 


\section{Antibody-Drug Conjugates}

Given the harsh side effects of chemotherapy, approaches that selectively target cytotoxic agents to cancer cells are an attractive strategy. This approach is very promising in the treatment of HER2/neu-positive cancer. In particular, trastuzumab-emtansine (T-DM1), an antibody-drug conjugate which has a chemotherapeutic agent linked to trastuzumab, has proven to be effective in treating tumors that had progressed on previous therapies, including trastuzumab therapy [48]. We have demonstrated that timing of chemotherapeutic administration can have different effects on the immune response initiated by anti-HER2/neu therapy [30••]. It will be important, therefore, to determine whether and to what extent trastuzumab-emtansine induces an immune response.

\section{Conclusion}

The discovery of the association between HER2/neu overexpression and breast cancer prognosis has promoted a succession of therapies aimed at exploiting this tumor oncoantigen, and the introduction of anti-HER2/neu antibody therapy has revolutionized the treatment of HER2positive breast cancer. Since approval of trastuzumab by the FDA, many studies have sought to understand the mechanisms by which this therapy reduces tumor burden. The recent identification that the adaptive immune response is necessary for mediating the effects of this antibody treatment has furthered the understanding of antiHER2/neu therapy and given credence to studies testing immunotherapies to fight HER2/neu-positive breast cancer. Further understanding the mechanisms behind current therapies, such as anti-HER2/neu antibody therapy, will benefit the development of these strategies.

Acknowledgments This research was supported in part by the National Institutes of Health grants CA141975 and CA97296 to Y.X.F.

Conflict of interest No potential conflicts of interest relevant to this article were reported.

\section{References}

Papers of particular interest, published recently, have been highlighted as:

- Of importance

-• Of major importance

1. Adams GP, Weiner LM (2005) Monoclonal antibody therapy of cancer. Nat Biotechnol 23(9):1147-1157
2. Griggs J, Zinkewich-Peotti K (2009) The state of the art: immune-mediated mechanisms of monoclonal antibodies in cancer therapy. Br J Cancer 101(11):1807-1812

3. Kruser TJ, Wheeler DL (2010) Mechanisms of resistance to HER family targeting antibodies. Exp Cell Res 316(7):1083-1100

4. Shih C, Padhy LC, Murray M, Weinberg RA (1981) Transforming genes of carcinomas and neuroblastomas introduced into mouse fibroblasts. Nature 290(5803):261-264

5. Padhy LC, Shih C, Cowing D, Finkelstein R, Weinberg RA (1982) Identification of a phosphoprotein specifically induced by the transforming DNA of rat neuroblastomas. Cell 28(4):865-871

6. Révillion F, Bonneterre J, Peyrat JP (1998) ERBB2 oncogene in human breast cancer and its clinical significance. Eur J Cancer 34(6):791-808

7. Rajkumar T, Gullick WJ (1994) The type I growth factor receptors in human breast cancer. Breast Cancer Res Treat 29(1): 3-9

8. Shepard HM, Brdlik CM, Schreiber H (2008) Signal integration: a framework for understanding the efficacy of therapeutics targeting the human EGFR family. J Clin Invest 118(11):3574-3581

9. Yarden Y, Pines G (2012) The ERBB network: at last, cancer therapy meets systems biology. Nat Rev Cancer 12(8):553-563

10. Quaglino E, Mastini C, Forni G, Cavallo F (2008) ErbB2 transgenic mice: a tool for investigation of the immune prevention and treatment of mammary carcinomas. Curr Protoc Immunol 82:20. 9.1-20.9.10

11. Ménard S, Pupa SM, Campiglio M, Tagliabue E (2003) Biologic and therapeutic role of HER2 in cancer. Oncogene 22(42): 6570-6578

12. Drebin JA, Link VC, Stern DF, Weinberg RA, Greene MI (1985) Down-modulation of an oncogene protein product and reversion of the transformed phenotype by monoclonal antibodies. Cell 41(3):697-706

13. Hudziak RM, Lewis GD, Winget M, Fendly BM, Shepard HM, Ullrich A (1989) p185HER2 monoclonal antibody has antiproliferative effects in vitro and sensitizes human breast tumor cells to tumor necrosis factor. Mol Cell Biol 9(3):1165-1172

14. Drebin JA, Link VC, Weinberg RA, Greene MI (1986) Inhibition of tumor growth by a monoclonal antibody reactive with an oncogene-encoded tumor antigen. Proc Natl Acad Sci USA 83(23):9129-9133

15. Hudziak RM, Lewis GD, Shalaby MR, Eessalu TE, Aggarwal BB, Ullrich A et al (1988) Amplified expression of the HER2/ ERBB2 oncogene induces resistance to tumor necrosis factor alpha in NIH 3T3 cells. Proc Natl Acad Sci USA 85(14): 5102-5106

16. Eccles SA (2001) Monoclonal antibodies targeting cancer: "magic bullets" or just the trigger? Breast Cancer Res 3(2):86-90

17. Carter P, Presta L, Gorman CM, Ridgway JB, Henner D, Wong WL et al (1992) Humanization of an anti-p185HER2 antibody for human cancer therapy. Proc Natl Acad Sci USA 89(10): 4285-4289

18. Nahta R, Esteva FJ (2006) Herceptin: mechanisms of action and resistance. Cancer Lett 232(2):123-138

19. Abramson V, Arteaga CL (2011) New strategies in HER2-overexpressing breast cancer: many combinations of targeted drugs available. Clin Cancer Res 17(5):952-958. doi:10.1158/10780432.CCR-09-1947

20. Slamon DJ, Leyland-Jones B, Shak S, Fuchs H, Paton V, Bajamonde A et al (2001) Use of chemotherapy plus a monoclonal antibody against HER2 for metastatic breast cancer that overexpresses HER2. New Eng1 J Med 344(11):783-792

21. Vogel C, Cobleigh M, Tripathy D, Gutheil J (2002) Efficacy and safety of trastuzumab as a single agent in first-line treatment of HER2-overexpressing metastatic breast cancer. J Clin Oncol 20(3): $719-726$ 
22. Cobleigh MA, Vogel CL, Tripathy D, Robert NJ, Scholl S, Fehrenbacher L et al (1999) Multinational study of the efficacy and safety of humanized anti-HER2 monoclonal antibody in women who have HER2-overexpressing metastatic breast cancer that has progressed after chemotherapy for metastatic disease. J Clin Oncol 17(9):2639-2648

23. Baselga J, Tripathy D, Mendelsohn J, Baughman S, Benz CC, Dantis L et al (1996) Phase II study of weekly intravenous recombinant humanized anti-p185HER2 monoclonal antibody in patients with HER2/neu-overexpressing metastatic breast cancer. J Clin Oncol 14(3):737-744

24. Hudis CA (2007) Trastuzumab - mechanism of action and use in clinical practice. N Engl J Med 357(1):39-51

25. Piccart-Gebhart MJ, Procter M, Leyland-Jones B, Goldhirsch A, Untch M, Smith I et al (2005) Trastuzumab after adjuvant chemotherapy in HER2-positive breast cancer. $\mathrm{N}$ Engl $\mathrm{J}$ Med 353(16):1659-1672

26. Romond EH, Perez EA, Bryant J, Suman VJ, Geyer CE, Davidson NE et al (2005) Trastuzumab plus adjuvant chemotherapy for operable HER2-positive breast cancer. N Engl J Med 353(16):1673-1684

27. Clynes RA, Towers TL, Presta LG, Ravetch JV (2000) Inhibitory $\mathrm{Fc}$ receptors modulate in vivo cytotoxicity against tumor targets. Nat Med 6(4):443-446

28. Raja S, Luan H, Naramura M, Bailey T, Clubb R, Band V et al (2011) Mechanisms of Trastuzumab resistance in ErbB2-driven breast cancer and newer opportunities to overcome therapy resistance. J Carcinog 10(1):28

29. Ladjemi MZ, Jacot W, Chardès T, Pèlegrin A, Navarro-Teulon I (2010) Anti-HER2 vaccines: new prospects for breast cancer therapy. Cancer Immunol Immunother 59(9):1295-1312

30. •• Park S, Jiang Z, Mortenson ED, Deng L, Radkevich-Brown O, Yang X et al (2010) The therapeutic effect of anti-HER2/neu antibody depends on both innate and adaptive immunity. Cancer Cell 18(2):160-170. This study is the first to identify the necessity of adaptive immunity in anti-HER2/neu therapy and the implications on current therapies.

31. Blander JM, Medzhitov R (2006) Toll-dependent selection of microbial antigens for presentation by dendritic cells. Nature 440(7085):808-812

32. Rakoff-Nahoum S, Medzhitov R (2009) Toll-like receptors and cancer. Nat Rev Cancer 9(1):57-63

33. Apetoh L, Ghiringhelli F, Tesniere A, Criollo A, Ortiz C, Lidereau $\mathrm{R}$ et al (2007) The interaction between HMGB1 and TLR4 dictates the outcome of anticancer chemotherapy and radiotherapy. Immunol Rev 220:47-59

34. •• Stagg J, Loi S, Divisekera U, Ngiow SF, Duret H, Yagita H et al (2011) Anti-ErbB-2 mAb therapy requires type I and II interferons and synergizes with anti-PD-1 or anti-CD137 mAb therapy. Proc Natl Acad Sci USA 108(17):7142-7147. This study highlights the critical role for type I and type II interferons and how anti-HER2/neu therapy may be enhanced by combination therapies such as anti-PD-1 therapy.

35. Capelan M, Pugliano L, de Azambuja E, Bozovic I, Saini KS, Sotiriou C et al (2012) Pertuzumab: new hope for patients with HER2-positive breast cancer. Ann Oncol. doi:10.1093/annonc/ mds 328

36. Gattinoni L, Powell DJ, Rosenberg SA, Restifo NP (2006) Adoptive immunotherapy for cancer: building on success. Nat Rev Immunol 6(5):383-393

37. Rosenberg SA (2005) The emergence of modern cancer immunotherapy. Ann Surg Oncol 12(5):344-346

38. Gajewski TF, Meng Y, Harlin H (2006) Immune suppression in the tumor microenvironment. J Immunother 29(3):233-240

39. Ryschich E, Schmidt J, Hämmerling GJ, Klar E, Ganss R (2002) Transformation of the microvascular system during multistage tumorigenesis. Int J Cancer 97(6):719-725

40. Buckanovich RJ, Facciabene A, Kim S, Benencia F, Sasaroli D, Balint $\mathrm{K}$ et al (2008) Endothelin B receptor mediates the endothelial barrier to $\mathrm{T}$ cell homing to tumors and disables immune therapy. Nat Med 14(1):28-36

41. Yu P, Lee Y, Liu W, Krausz T, Chong A, Schreiber H et al (2005) Intratumor depletion of CD4+ cells unmasks tumor immunogenicity leading to the rejection of late-stage tumors. J Exp Med 201(5):779-791

42. Rabinovich GA, Gabrilovich D, Sotomayor EM (2007) Immunosuppressive strategies that are mediated by tumor cells. Annu Rev Immunol 25:267-296

43. - Vanneman M, Dranoff G (2012) Combining immunotherapy and targeted therapies in cancer treatment. Nat Rev Cancer 12(4):237-251. This review thoroughly covers immunotherapy in breast cancer as well as other cancer types.

44. Boon T, van der Bruggen P (1996) Human tumor antigens recognized by T lymphocytes. J Exp Med 183(3):725-729

45. Paul WE (ed) (1999) Fundamental immunology, 4th edn. Lippincott Williams \& Wilkins, Philadelphia

46. Kohrt HE, Houot R, Weiskopf K, Goldstein MJ, Scheeren F, Czerwinski D et al (2012) Stimulation of natural killer cells with a CD137-specific antibody enhances trastuzumab efficacy in xenotransplant models of breast cancer. J Clin Invest 122(3): $1066-1075$

47. Yu P, Lee Y, Wang Y, Liu X, Auh S, Gajewski TF et al (2007) Targeting the primary tumor to generate CTL for the effective eradication of spontaneous metastases. J Immunol 179(3): 1960-1968

48. Murphy CG, Morris PG (2012) Recent advances in novel targeted therapies for HER2-positive breast cancer. Anticancer Drugs 23(8):765-776 